\title{
Normalization of Mendeley reader counts for impact assessment
}

\author{
Robin Haunschild* and Lutz Bornmann ${ }^{* *}$
}

\author{
*First author and corresponding author: \\ Max Planck Institute for Solid State Research \\ Heisenbergstr. 1, \\ 70569 Stuttgart, Germany. \\ Email: R.Haunschild@fkf.mpg.de
}

** Contributing author:

Division for Science and Innovation Studies

Administrative Headquarters of the Max Planck Society

Hofgartenstr. 8,

80539 Munich, Germany.

Email: bornmann@gv.mpg.de 


\begin{abstract}
A different number of citations can be expected for publications appearing in different subject categories and publication years. For this reason, the citation-based normalized indicator Mean Normalized Citation Score (MNCS) is used in bibliometrics. Mendeley is one of the most important sources of altmetrics data. Mendeley reader counts reflect the impact of publications in terms of readership. Since a significant influence of publication year and discipline has also been observed in the case of Mendeley reader counts, reader impact should not be estimated without normalization. In this study, all articles and reviews of the Web of Science core collection with a publication year of 2012 (and a DOI) are used to normalize their Mendeley reader counts. A new indicator that determines the normalized reader impact is obtained — the Mean Normalized Reader Score (MNRS) - and compared with the MNCS. The MNRS enables us to compare the impact a paper has had on Mendeley across subject categories and publication years.

Comparisons on the journal and university level show that the MNRS and MNCS correlate larger for 9,601 journals than for 76 German universities.
\end{abstract}

\title{
Key words
}

Altmetrics, Mendeley, normalization, reader impact 


\section{Introduction}

Estimating the citation impact of scientists, research groups, and institutes in different disciplines and time periods faces the problem that discipline and time period influence the citation impact of publications independently of the quality of the publications. Normalization for both factors started in the mid-1980s (Schubert \& Braun, 1986). Only since normalized values were obtained did it become possible to assess the citation impact of entities such as researchers or universities across disciplines and time periods. In the calculation of a normalized impact value for a publication, the total number of citations of the publication is counted (times cited). The number of times cited is compared with the citation impact of publications with the same publication year, subject category, and document type (expected impact of the reference set). This technique is referred to as cited-side normalization. Although other methods have been developed in recent years (e.g., normalization on the side of the citing publications, Zitt \& Small, 2008), this method is the most established and used in bibliometrics.

In recent years, impact evaluation in scientometric research has been done not only on the basis of citations but also based on alternative metrics (altmetrics) (Borrego, 2014; Mohammadi \& Thelwall, 2014; Torres-Salinas, Cabezas-Clavijo, \& Jimenez-Contreras, 2013; Priem, 2013; Priem, 2014). Altmetrics open the possibility to assess the impact of research faster than with citations. Moreover, altmetrics seem suitable to determine the impact of research in a broader manner than with citations (Aguinis, Shapiro, Antonacopoulou, \& Cummings, 2014; Bar-Ilan et al., 2012; Bornmann, 2014; Dinsmore, Allen, \& Dolby, 2014; Hammarfelt, 2014; Priem, Taraborelli, Groth, \& Neylon, 2010). While citations quantify only the impact of research on science, altmetrics could be able to quantify the impact of research on all aspects of society, including science. Current scientometric research studies if this hope is more than a working hypothesis. 


\section{Literature overview and research questions}

Data from Mendeley are among the most important sources for altmetrics: "Mendeley is both a citation management tool and social network for scholars with over two million users" (Rodgers \& Barbrow, 2013, p. 12). One basic assumption behind the use of such data in an evaluative context is that Mendeley readers who add a publication to their library can be counted as readers of the publication. Indeed, the results of Mohammadi, Thelwall, and Kousha (in press) show that " $82 \%$ of the Mendeley users had read or intended to read at least half of the bookmarked publications in their personal libraries." Therefore, Mendeley counts are seen as a very promising possibility to quantify the size of the readership of a paper inside as well as outside of science. Furthermore, a Mendeley reader can be seen as a precursor to a citer, as Mendeley users include a publication into their library when they intend to cite it in a forthcoming manuscript. However, each Mendeley user is counted as one reader, while it is possible that they will cite the publication multiple times or not at all.

Several studies have shown that the Mendeley reader impact-similar to the citation impact, although there are differences between the two- - varies across scientific disciplines (Jeng, He, \& Jiang, 2015; Thelwall \& Maflahi, 2015; Zahedi, Costas, \& Wouters, 2014; Zahedi \& Eck, 2014). In one discipline, papers are read more often on average (or papers are more frequently included in the user's Mendeley library) than in other disciplines. These variations are not only specific to Mendeley data but also to other altmetric sources, e.g., Twitter counts (Haustein, Costas, \& Lariviere, 2015). Moreover, publications with different document types and publication years receive different average numbers of Mendeley readers (Haustein \& Lariviere, 2014). Therefore, in almost the same manner as for citation counts, Mendeley reader counts should be normalized with respect to publication year, document type, and scientific discipline before an interpretation is attempted. 
The aim of this study is to apply the most established method of normalization (cited-side) in bibliometrics to the field of altmetrics and propose a normalization scheme for Mendeley reader counts. Independently from and coincidental with our efforts (Haunschild \& Bornmann, 2015) a similar approach has been suggested by Fairclough \& Thelwall, 2015, which focuses on country comparisons only. The possibility of defining an indicator similar to the MNCS but based on reader counts instead of citations constitutes our first research question. The second research question addresses to which extent the MNCS correlates with the indicator based on reader counts on the journal and university levels.

\section{Data Set}

It is common practice in scientometrics to evaluate the impact of articles and reviews. Other document types are usually not included in evaluative bibliometrics (Moed, 2005). We retrieved the Mendeley reader statistics for articles and reviews published in 2012 and having a DOI $\left(n_{\mathrm{A}}=1,133,224\right.$ articles and $n_{\mathrm{R}}=64,960$ reviews) via the Mendeley API made available in 2014. The DOIs of the papers from 2012 were exported from the in-house database of the Max Planck Society (MPG) based on the WoS and administered by the Max Planck Digital Library (MPDL). We used R (http://www.r-project.org/) to interface to the Mendeley API. DOIs were used to identify papers in the Mendeley API; 1,074,407 articles (94.8\%) and 62,771 reviews (96.6\%) were found at Mendeley.

In total, the articles were registered in Mendeley 9,352,424 times and the reviews were registered 1,335,764 times. For 118,167 articles (10.4\%) and 4,348 reviews (6.7\%), we found the paper at Mendeley but without a reader. Papers without any readers indexed by Mendeley may originate from former readers who removed the paper from their library or closed their Mendeley account. If Mendeley users include too little bibliographic data for a paper in their library, they 
are not counted as readers either, because there is insufficient information to link them to a Mendeley database entry. Also, Mendeley adds papers to the database without any reader in the first place from publisher feeds. Therefore, papers with zero reader counts should be excluded in this study, or, if they are included, the papers not found at Mendeley should also be counted as papers with zero readers. We tested both approaches and found no significant differences regarding the scope of this study. In the end, we decided to include the papers with zero readers as well as the papers we did not find in the Mendeley API. This is consistent with the way citations are handled in bibliometric databases. The requests to the Mendeley API were made from December 11-23, 2014. All data in this study are based on a partial copy of our in-house database (last updated on November 23, 2014) supplemented with the Mendeley reader counts.

\section{Results}

\subsection{Differences in reader impact between subject categories}

Like the citation distribution (Albarran, Crespo, Ortuno, \& Ruiz-Castillo, 2011;

Rodriguez-Navarro, 2011; Seglen, 1992), the reader distribution is skewed across subject categories, as shown in Figure 1 for articles and Figure 2 for reviews. 


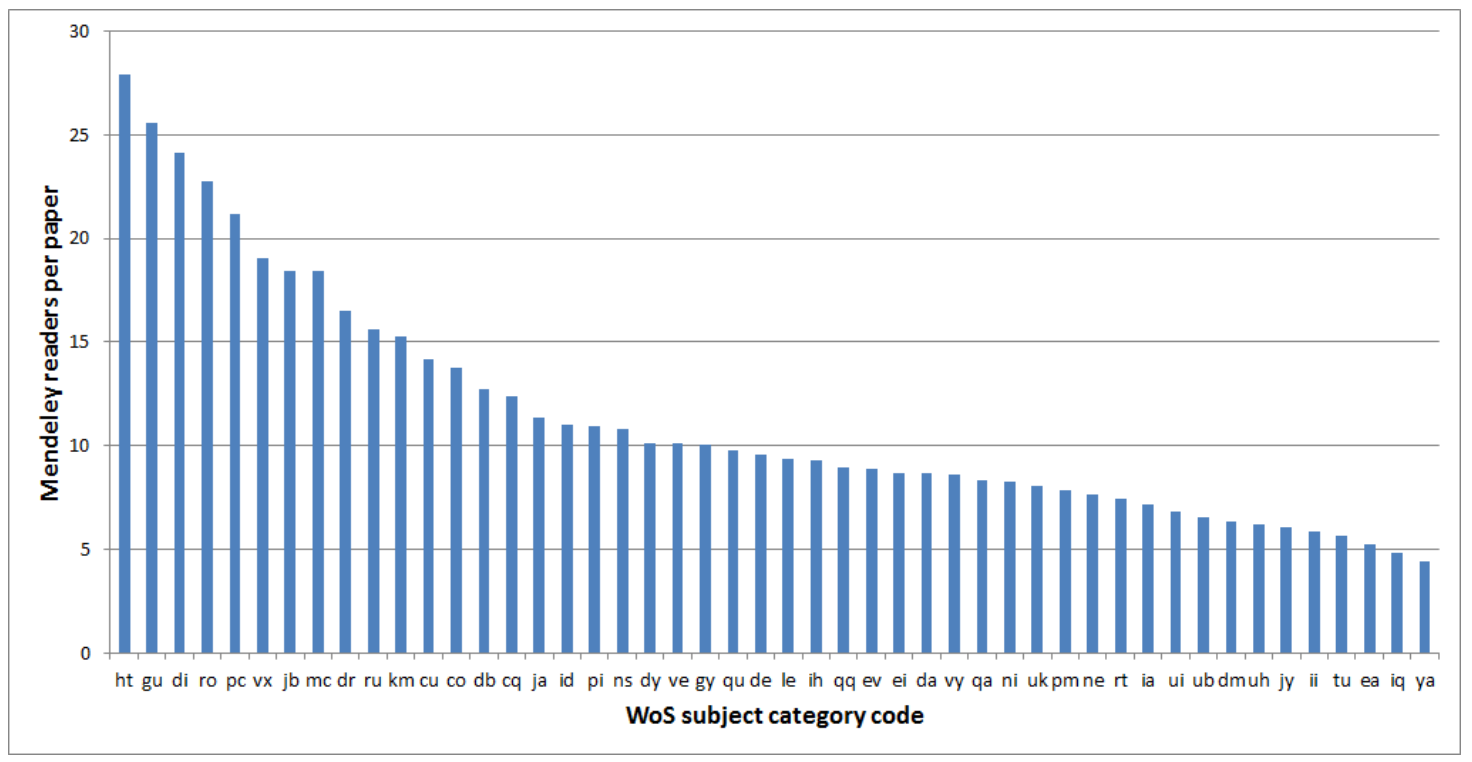

Fig. 1: Distribution of Mendeley readers per paper across WoS subject categories for the document type article. Only subject categories including papers with articles registered in Mendeley at least 90,000 times in total are shown. A table with all the WoS subject categories and the explanation of the two-letter codes is located in the Appendix.

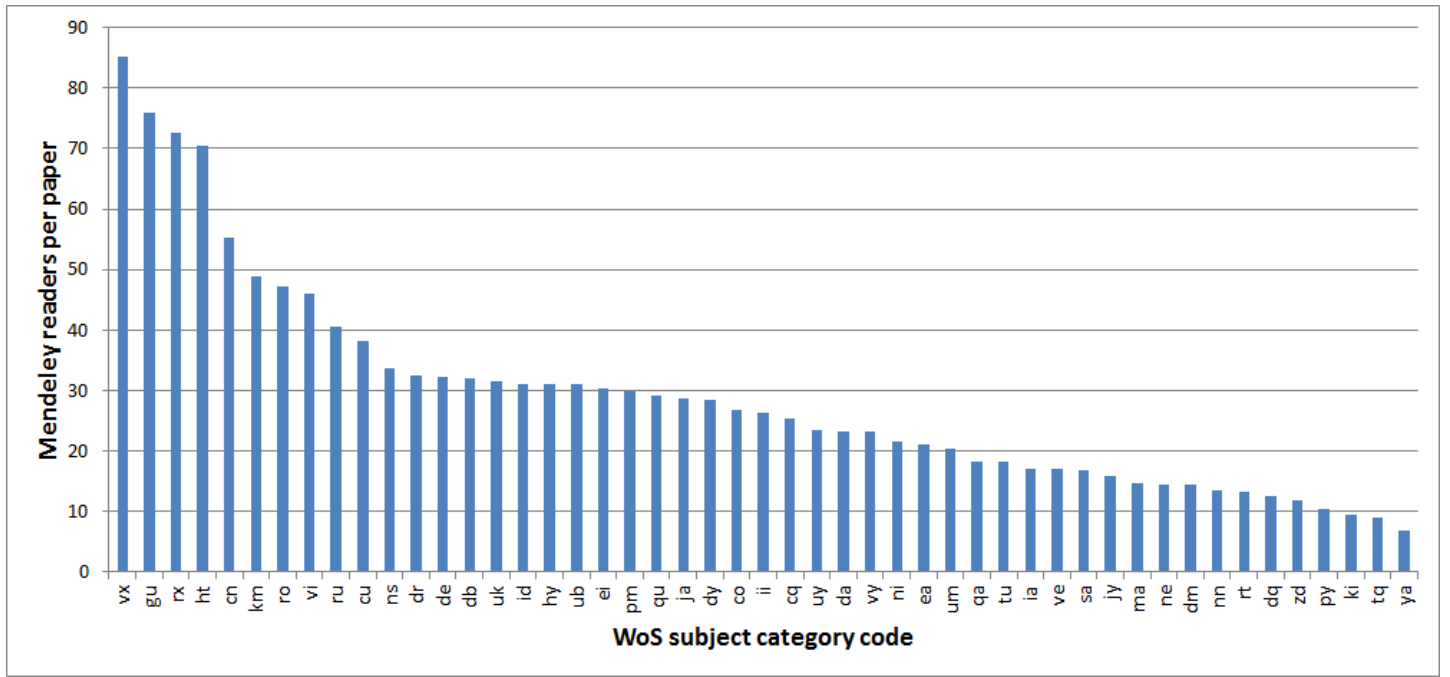

Fig. 2: Distribution of Mendeley readers per paper across WoS subject categories for the document type review. Only subject categories including papers with reviews registered in Mendeley at least 10,000 times in total are shown. A table with all the WoS subject categories and the explanation of the two-letter codes is located in the Appendix.

The reader distribution across the categories ranges from 0.22 readers per paper in Poetry (not shown in Fig. 1; see Table A.1 in the Appendix) to 27.94 readers per paper in Evolutionary 
Biology (WoS category "ht" in Fig. 1). The 30 most highly populated WoS categories (12\% of the 251 categories) comprise $50 \%$ of the readership of the articles studied here.

For reviews, the highest number of readers per paper (85.22) is found in the WoS category Psychology, Experimental (WoS category "vx" in Fig. 2), while the lowest (non-zero) number (0.27) is in Literature (not shown in Fig. 2; see Table A.1 in the Appendix). Fifty percent of the readers are found in the 18 ( $7.5 \%$ of the 239 categories) most highly populated WoS categories.

Usually, reviews are cited more often than articles. This seems to hold true for Mendeley reader counts. Figures 1 and 2 as well as Table A.1 in the Appendix show that reviews are also read more often than articles, on average. The overall average value of readers per paper is 8.25 for articles and 20.56 for reviews. This shows the need for a normalization of Mendeley reader counts with respect to subject categories and document types. Thus, the normalization procedure is done separately for articles and reviews. A more general discussion about the WoS document types can be found elsewhere (Harzing, 2013).

\subsection{Normalization of reader impact}

For normalization purposes in bibliometrics, the citation impact of a focal paper is compared with the expected citation impact. The expected value is the average citation impact in the same discipline, publication year, and document type as the paper in question. Sometimes, publications of different document types are considered together in the calculation of the expected value (as in the Leiden Ranking) or separately (as in InCites from Thomson Reuters, SciVal from Elsevier, and the Institutions Ranking published by the SCImago group). The paper set determining the expected value is referred to as the reference set. The ratio of observed and expected citations is the Normalized Citation Score (NCS). Currently, the NCS is the established standard in bibliometrics to normalize citation impact. A NCS of 1 for a publication indicates an 
average citation impact. A NCS of 1.5 can be interpreted as a citation impact that is $50 \%$ higher than the average (Waltman, van Eck, van Leeuwen, Visser, \& van Raan, 2011a; Waltman, van Eck, van Leeuwen, Visser, \& van Raan, 2011b).

If a paper has been assigned (e.g., by a database provider such as Thomson Reuters) to more than one subject category, the average value of all NCS values is used (resulting in a mean NCS). The assignment of papers to multiple subjects leads to an average value over all papers of a publication year that differs from 1 . To alleviate this disadvantage, one can employ fractional counting (Waltman \& Eck, 2014), multiplicative counting (Herranz \& Ruiz-Castillo, 2012), or full counting with a scaling of all NCS values (Haunschild \& Bornmann, submitted). We decided to use the multiplicative counting method, although other counting methods could be used, too. We do not expect different conclusions of our study if other reasonable counting methods were to be used.

In the case of aggregations of papers (normalized impact of a researcher or university), the average value of the NCS values of all papers in the set is calculated. This average value is referred to as the Mean Normalized Citation Score (MNCS). The MNCS is used in the SCImago Institutions Ranking (SCImago Research Group, 2013) (in SCImago Institutions Ranking, the MNCS is referred to as the Normalized Impact ${ }^{1}$ ), InCites ${ }^{2}$, and in the Times Higher Education Ranking (THE Ranking ${ }^{3}$ ).

Following the definition of the MNCS, we propose a Mean Normalized Reader Score (MNRS) using the multiplicative counting method for papers categorized to multiple subjects. Our normalization procedure for Mendeley reader impact starts with the calculation of the

\footnotetext{
${ }^{1}$ See http://www.scimagoir.com/methodology.php

${ }^{2} \mathrm{See}$ http://researchanalytics.thomsonreuters.com/m/pdfs/indicators-handbook.pdf

${ }^{3} \mathrm{See}$ https://www.timeshighereducation.com/world-university-rankings-2014-15-methodology
} 
average number of Mendeley readers per paper $\left(\rho_{c}\right)$ in each WoS category (cf. Fig. 1 and Table A.1 in the Appendix):

$$
\rho_{c}=\frac{1}{N_{c}} \sum_{i=1}^{N_{c}} R_{i c}
$$

Here, $R_{i c}$ denotes the raw Mendeley reader count of paper $i$, which has been assigned to WoS category $c$, and $N_{c}$ is the number of papers assigned to WoS category $c$. Afterwards, the raw Mendeley reader count is divided by the average number of Mendeley readers per paper in WoS category $c\left(\rho_{c}\right)$, yielding the normalized reader score (NRS) for paper $i$ in subject category $c$ :

$$
N R S_{i c}=\frac{R_{i c}}{\rho_{c}}
$$

The average value over all NRS values equals exactly one (due to the multiplicative counting method).

Since we include only papers that were published in 2012, the publication year does not have to be included in the normalization procedure. The overall reader impact of a specific aggregation level (e.g., researcher, institute, or country) can be analyzed on the basis of the mean value over the paper set. This results in the mean NRS (MNRS) for the paper set.

As an illustrative example, we show a step by step calculation of the NRS for the article with DOI 10.1061/(asce)co.1943-7862.0000464. We recorded a reader count of 8 for this article. The article is classified in the WoS subject categories Constructions \& Building Technology (fa), Engineering, Civil (im), and Engineering, Industrial (ij). The average reader counts for these WoS subject categories are 9.30, 7.51, and 11.69, respectively. Therefore, we obtain $\mathrm{NRS}_{\mathrm{fa}}=$ $0.86, \mathrm{NRS}_{\mathrm{im}}=1.07$, and $\mathrm{NRS}_{\mathrm{ij}}=0.68$. This paper has a reader impact slightly above average in the category "im" but below average in the categories "fa" and "ij." Using the multiplicative 
counting method, papers assigned to multiple categories do not have a single impact value. For example, if this paper belongs to the publication set of a country and the MNRS is calculated for this set, the paper is not considered only once but three times (with potentially different impact values in each subject category) in the calculation of the average NRS value.

Table 1 shows the thresholds of NRS values that a paper has to have at least in order to become a top $1 \%$ and top $10 \%$ paper. The differences between the NRS thresholds for reviews and articles are smaller for the top $10 \%$ than for the top $1 \%$.

Table 1: NRS thresholds for top $1 \%$ and top $10 \%$ papers

\begin{tabular}{lcc}
\hline & \multicolumn{2}{c}{ NRS threshold } \\
\cline { 2 - 3 } & Articles & Reviews \\
\hline Top 1\% & 7.11 & 6.72 \\
Top 10\% & 2.43 & 2.39 \\
\hline
\end{tabular}

Normalized readership values can be calculated for different units: single researchers, research groups, institutions, countries, journals, etc. As examples, we present in the following the MNRS values for journals and several German universities.

\subsection{Normalized reader impact of journals}

Papers from 9,601 journals out of the 12,334 WoS journals in 2012 are covered in the papers found at Mendeley. Table 2 shows the 25 journals with at least 400 papers in 2012 and highest MNRS values. The minimum of 400 unique papers published in 2012 ensures the calculation of reliable journal MNRS values on a broad paper basis. Many reputable journals (Cell, Nature, and Science) are also journals with high MNRS values. For example, Cell has an MNRS of 10.03. This means that papers published in Cell have been read on average ten times as 
often as papers in the corresponding reference sets (papers published in the same subject categories). Papers published in Solar Energy Materials and Solar Cells also have more readers on average than one can expect. However, these papers are only read twice as often on average as papers in the corresponding subject categories. We have included the MNCS in addition to the MNRS in Table 2 (the MNCS is also based on the multiplicative counting method and the same data set as the MNRS).

Table 2: Top 25 WoS journals with the highest MNRS values in 2012 ordered by decreasing MNRS (only journals with a minimum of 400 unique papers are considered)

\begin{tabular}{lllll}
\hline Journal & $\begin{array}{c}\text { No. of papers } \\
\text { (multiplicative } \\
\text { counting) }\end{array}$ & $\begin{array}{c}\text { No. of papers } \\
\text { (unique papers) }\end{array}$ & MNRS & MNCS \\
\hline Cell & 830 & 415 & 10.03 & 6.89 \\
Nature & 869 & 869 & 7.14 & 8.13 \\
Science & 830 & 830 & 4.82 & 5.40 \\
Nano Letters & 6,462 & 1,077 & 4.69 & 3.46 \\
Advanced Materials & 5,202 & 867 & 3.20 & 3.50 \\
ACS Nano & 4,764 & 1,191 & 3.13 & 2.71 \\
Journal of the American College of & 426 & 426 & 2.91 & 3.56 \\
Cardiology & & & & \\
Physical Review Letters & 3,789 & 3,789 & 2.85 & 2.33 \\
PLoS Computational Biology & 1,014 & 507 & 2.82 & 1.65 \\
Cancer Research & 625 & 625 & 2.82 & 2.11 \\
Circulation & 1,236 & 618 & 2.80 & 3.34 \\
Plant Physiology & 469 & 469 & 2.66 & 2.71 \\
Energy and Environmental Science & 1,892 & 473 & 2.63 & 4.79 \\
NeuroImage & 3,663 & 1,221 & 2.62 & 1.71 \\
Bioinformatics & 2,184 & 728 & 2.57 & 1.48 \\
Journal of Medicinal Chemistry & 889 & 889 & 2.25
\end{tabular}




\begin{tabular}{lllll} 
Nucleic Acids Research & 1,425 & 1,425 & 2.43 & 2.33 \\
$\begin{array}{l}\text { Journal of the American Chemical } \\
\text { Society }\end{array}$ & 3,095 & 3,095 & 2.43 & 2.24 \\
PLoS Pathogens & 1,881 & & & \\
Analytical Chemistry & 1,479 & 627 & 2.39 & 1.99 \\
Pediatrics & 686 & 1,479 & 2.38 & 2.03 \\
Advanced Functional Materials & 3,414 & 686 & 2.34 & 2.53 \\
Molecular Ecology & 1,335 & 569 & 2.30 & 2.61 \\
Journal of Neuroscience & 1,667 & 445 & 2.30 & 1.51 \\
Solar Energy Materials and Solar Cells & 1,476 & 1,667 & 2.20 & 1.73 \\
\hline
\end{tabular}

The MNRS of all 9601 journals correlates much larger than typical with the MNCS, which is indicated by the Spearman rank coefficient of 0.70 (for the interpretation of correlation coefficients, see Cohen, 1988 and Kraemer et al., 2003). A scatter plot of the MNCS and MNRS values for 9,601 WoS journals is shown in Figure 3. Any comparison of the MNRS and MNCS values has to be done carefully, as citations do not aggregate as quickly as Mendeley reader counts. Papers published in 2012 have had a short citation window, as citations were gathered for this study until November 23, 2014. 


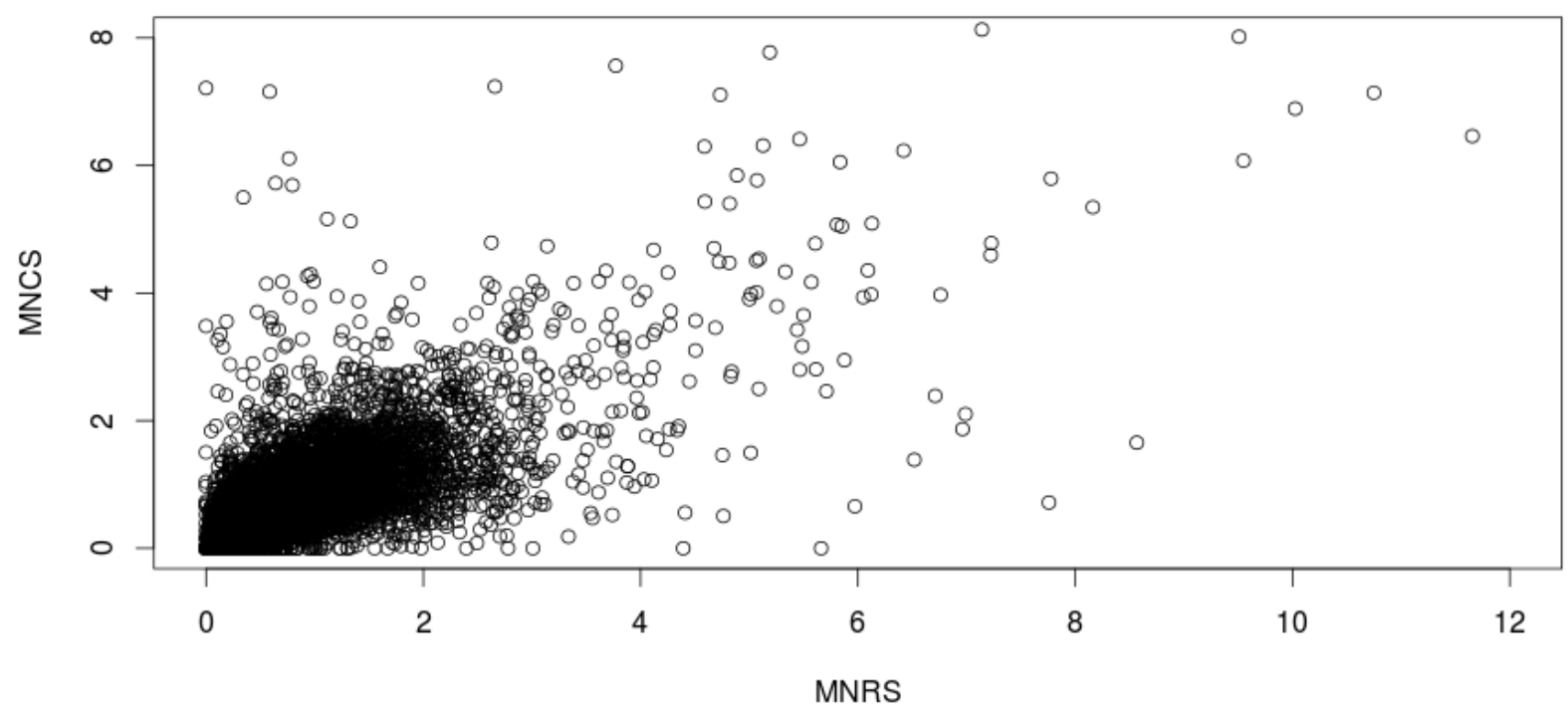

Fig. 3: Scatter plot of MNCS and MNRS values for 9,601 WoS journals

\subsection{Normalized reader impact of universities}

Table 3 shows the MNCS and MNRS values as well as papers published in 2012 for the top 10 German universities according to the Leiden Ranking 2013

(http://www.leidenranking.com/ranking/2013) ordered by decreasing MNRS. The indicatorspecific values of all universities are similar and above the average reader impact of $\mathrm{MNRS}=1$ :

The range is from $21 \%$ to $46 \%$ above average in their corresponding subject categories. The MNCS value is consistently higher than the MNRS value for the universities in Table 3.

Although the MNRS values of the top 10 German universities indicate that the universities perform up to $46 \%$ better than average, their MNRS average values are still quite far away from the top $10 \%$ and top $1 \%$ thresholds of individual papers: top 10\%: 2.43 for articles and 2.39 for reviews; top 1\%: 7.11 for articles and 6.72 for reviews (see also Table 1). 
Table 3: Top 10 German universities with MNCS, MNRS, and papers published in 2012 (unique and multiplicative counting), ordered by decreasing MNRS

\begin{tabular}{lcccc}
\hline University & MNCS & MNRS & $\begin{array}{c}\text { Papers in 2012 } \\
\text { (unique paper } \\
\text { counting) }\end{array}$ & $\begin{array}{c}\text { Papers in 2012 } \\
\text { (multiplicative } \\
\text { counting) }\end{array}$ \\
\hline TU Munich & 1.51 & 1.46 & 3,399 & 7,033 \\
LMU Munich & 1.62 & 1.42 & 4,500 & 9,214 \\
University Würzburg & 1.62 & 1.41 & 2,035 & 3,883 \\
University Freiburg & 1.45 & 1.40 & 2,582 & 5,819 \\
University Göttingen & 1.62 & 1.34 & 2,528 & 4,819 \\
Heidelberg University & 1.54 & 1.30 & 3,721 & 7,625 \\
University Bonn & 1.64 & 1.26 & 2,780 & 5,350 \\
University Münster & 1.46 & 1.26 & 2,210 & 4,803 \\
RWTH Aachen & 1.54 & 1.22 & 2,539 & 5,732 \\
Goethe University Frankfurt & 1.49 & 1.21 & 2,341 & 4,607 \\
\hline
\end{tabular}

A broader comparison of MNCS and MNRS can be performed when all German universities with at least 100 papers in 2012 are included. The MNRS correlates slightly larger than typical with the MNCS in this case, as indicated by the Spearman rank coefficient of 0.41 (for the interpretation of correlation coefficients, see Cohen, 1988 and Kraemer et al., 2003). A scatter plot of the MNCS and MNRS values for the 76 German universities is shown in Figure 4. It is to be expected that the correlation between MNRS and MNCS is higher for journals (see section 4.3) than for universities because the MNRS and MNCS are normalized with respect to WoS subject categories which are journal sets. 


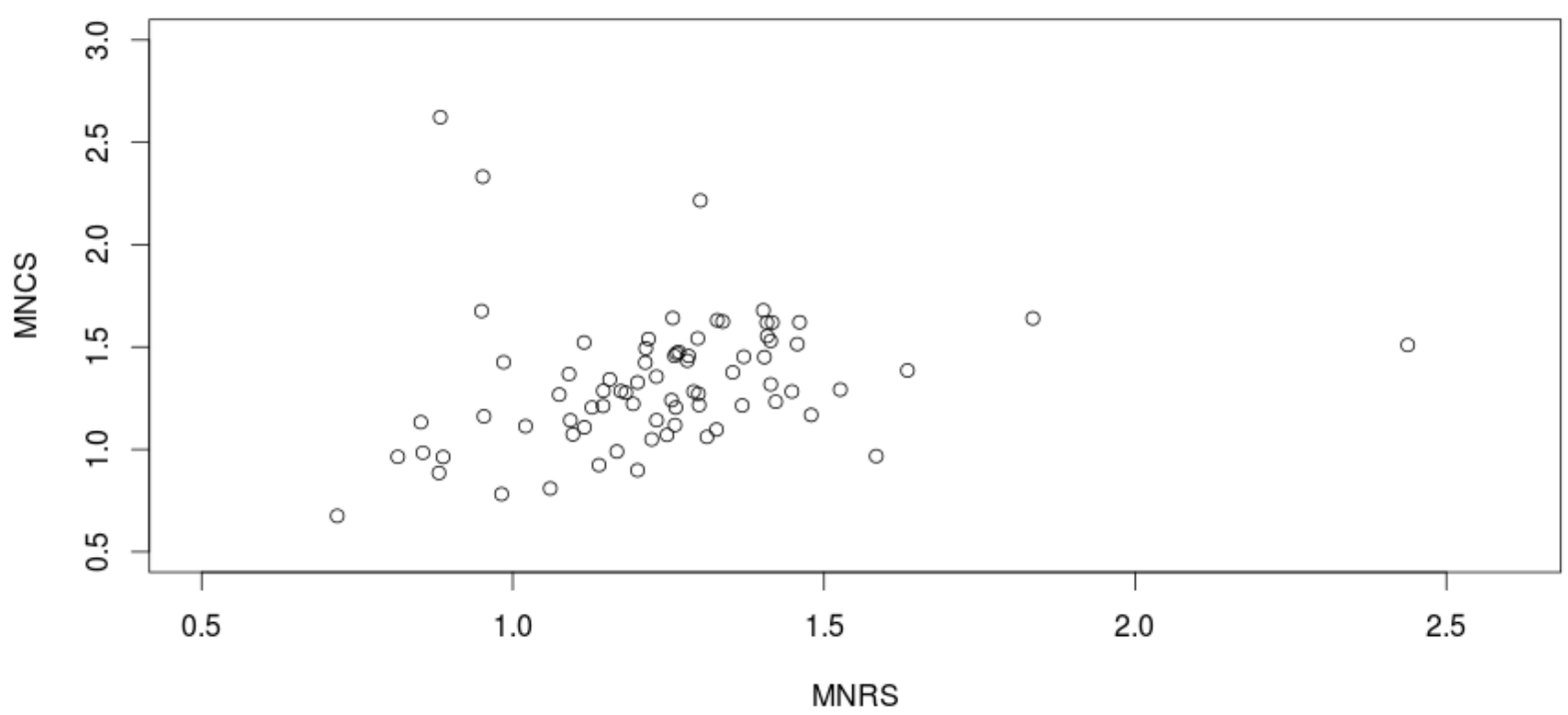

Fig. 4: Scatter plot of MNCS and MNRS values for 76 German universities

\section{Discussion}

The Directorate for Science, Technology, and Innovation in Brussels stated that the "use of indicators of social media is relatively well established in advertising and marketing. Some organisations are starting to collect altmetrics on a commercial basis in relation to research publications. In this context, little is known about what individual altmetrics mean. This appears to provide a rich set of opportunities for research - though we are probably some time away from being able to think in a precise way about concepts such as a 'field-normalised tweet'" (Committee for Scientific and Technological Policy, 2014, p. 43). Here we proposed a fieldnormalized indicator based on altmetrics. Successful applications for journal and university rankings have been shown. The MNRS values can also be applied for individual researchers and other aggregation units. Although we used Mendeley readership counts, a normalization procedure similar to the one used for MNRS can also be performed for tweets and other types of altmetrics. 
We found a high correlation between the MNCS and MNRS values for the ranking of journals. The MNRS values can be interpreted (analogously to MNCS values) such that a value of 1.5 indicates that a paper has $50 \%$ more Mendeley readers than an average paper in the same subject category. Following the rules of thumb by van Raan (2005) for citation-based indicators, an MNRS between 0.8 and 1.2 should be regarded as an average impact, while one above 1.2 and below 0.8 should be regarded as above and below average impact, respectively.

In addition to normalization methods which are based on average reader scores (MNRS), one can also use percentile-based approaches. Percentile-based approaches produce more robust normalized scores because they are not based on average values (of citations or readers) (Wilsdon et al., 2015). The percentile impact is the proportion of papers in a reference set that are cited or read, respectively, equal to or less often than the paper in question (Bornmann, Leydesdorff, \& Mutz, 2013). We made one step in the direction of calculating percentiles by determining the MNRS values that are needed for a paper to be among the top $1 \%$ and top $10 \%$ most-read papers.

What are the limitations of our study? One can see our retrieval strategy on Mendeley reader counts via the API using the DOI as a limitation. However, as we found the vast majority of papers (94.8\% of articles and $96.6 \%$ of reviews) with this method, we expect no major influence on our results from this retrieval strategy. Another limitation of our study is the exclusion of articles and reviews without a DOI. This reduces the number of publications from $1,390,504$ to $1,198,184$. Therefore, $86.2 \%$ of the articles and reviews of the WoS core collection of 2012 are included in this study. Under the assumption that publications with and without a DOI are evenly distributed across high and low impact publications, this will not alter our results significantly. This study is not intended to provide reader counts as reference values for later use. The main aim is to explore an established method from bibliometrics in the realm of altmetrics 
and to propose a method to normalize Mendeley reader counts in order to judge the reader impact of individual publications as well as aggregates of publications.

The normalization procedure proposed in this study can in principle also be applied to other altmetrics data, such as tweets and blog posts, as it relies on an external classification system assigned to individual papers (or journals where the paper was published). However, normalization with respect to other altmetrics data requires that a large proportion of the publication set is covered by the specific type of altmetrics source. This is the case for Mendeley reader counts, but this might not be the case for other sources of altmetrics data.

The interpretation of Mendeley reader counts seems to be more problematic than the interpretation of citation counts. Many scientists do not read papers in the Mendeley application or on the web interface. Often, scientists add a paper to their Mendeley library when they intend to read it. Although there are reasons to include a paper in one's Mendeley library other than reading it later, it has been proposed to interpret Mendeley reader counts as the number of citers to be (see above).

\section{Conclusions}

In this study, we have proposed a method for the normalization of Mendeley reader counts that is based on an established method of normalization for citation counts. We have shown that this method is able to normalize Mendeley reader counts. Comparisons on the journal and university level show that the MNRS and MNCS correlate larger for 9,601 journals than for 76 German universities.

Since the MNRS has been derived from a well-known and accepted variant used in bibliometrics, the conceptual justification of the new indicator seems to be given. However, further large-scale empirical investigations are necessary. We encourage other researchers in 
scientometrics to calculate MNRS values for different research units (journals, researchers, institutions, etc.) in order to test the reliability and validity of this indicator (e.g., by comparing the MNRS with other performance indicators).

The calculation of the MNRS needs further data besides data from Mendeley in order to have a field-classification scheme for normalization. In this study, we used the WoS subject categories as classification scheme. Since Mendeley users classify themselves in scientific disciplines (Haunschild, Bornmann, \& Leydesdorff, 2015) the Mendeley classification scheme could in principle be used for normalization, too. However, the use of the Mendeley classification scheme would entail the transfer of another kind of bibliometric normalization to Mendeley reader counts which works on the citing-side. In the citing-side normalization every citation of a paper is normalized with respect to its subject category (Bornmann \& Marx, 2015). The transfer of the citing-side bibliometric normalization to Mendeley reader counts is not as straightforward as the transfer of the cited-side bibliometric normalization and therefore beyond the scope of our present study. 


\section{Acknowledgements}

The bibliometric data used in this paper are from an in-house database developed and maintained by the Max Planck Digital Library (MPDL, Munich) and derived from the Science Citation Index Expanded (SCI-E), Social Sciences Citation Index (SSCI), and Arts and Humanities Citation

Index (AHCI) prepared by Thomson Reuters (Philadelphia, Pennsylvania, USA). The Mendeley reader counts were retried via the Mendeley API. 


\section{References}

Aguinis, H., Shapiro, D. L., Antonacopoulou, E. P., \& Cummings, T. G. (2014). Scholarly Impact: A Pluralist Conceptualization. Academy of Management Learning \& Education, 13(4), 623-639. doi: 10.5465/amle.2014.0121.

Albarran, P., Crespo, J. A., Ortuno, I., \& Ruiz-Castillo, J. (2011). The skewness of science in 219 sub-fields and a number of aggregates. Scientometrics, 88(2), 385-397. doi: 10.1007/s11192-011-0407-9.

Bar-Ilan, J., Haustein, S., Peters, I., Priem, J., Shema, H., \& Terliesner, J. (2012). Beyond citations: scholars'visibility on the social Web. Paper presented at the 17th International Conference on Science and Technology Indicators, Montreal, Quebec: Science-Metrix and OST.

Bornmann, L. (2014). Do altmetrics point to the broader impact of research? An overview of benefits and disadvantages of altmetrics. Journal of Informetrics, 8(4), 895-903. doi: 10.1016/j.joi.2014.09.005.

Bornmann, L., Leydesdorff, L., \& Mutz, R. (2013). The use of percentiles and percentile rank classes in the analysis of bibliometric data: opportunities and limits. Journal of Informetrics, 7(1), 158-165.

Bornmann, L., \& Marx, W. (2015). Methods for the generation of normalized citation impact scores in bibliometrics: Which method best reflects the judgements of experts? Journal of Informetrics, 9(2), 408-418.

Borrego, A. (2014). Altmetrics for the research evaluation and the analysis of the needs of information. Profesional De La Informacion, 23(4), 352-357. doi: 10.3145/epi.2014.jul.02.

Cohen, J. (1988). Statistical power analysis for the behavioral sciences (2nd ed.). Hillsdale, NJ, USA: Lawrence Erlbaum Associates, Publishers.

Committee for Scientific and Technological Policy. (2014). Assessing the impact of state interventions in research-techniques, issues and solutions. Brussels, Belgium: Directorate for Science, Technology and Innovation, Committee for Scientific and Technological Policy.

Dinsmore, A., Allen, L., \& Dolby, K. (2014). Alternative Perspectives on Impact: The Potential of ALMs and Altmetrics to Inform Funders about Research Impact. Plos Biology, 12(11). doi: 10.1371/journal.pbio.1002003.

Fairclough, R. \& Thelwall, M. (2015). National research impact indicators from Mendeley readers. Journal of Informetrics, 9(4), 845-859.

Hammarfelt, B. (2014). Using altmetrics for assessing research impact in the humanities. Scientometrics, 101(2), 1419-1430. doi: 10.1007/s11192-014-1261-3.

Harzing, A.-W. (2013). Document categories in the ISI Web of Knowledge: Misunderstanding the Social Sciences? Scientometrics, 94(1), 23-34. doi 10.1007/s11192-012-0738-1.

Haunschild, R., \& Bornmann, L. (submitted). The proposal of using scaling for calculating fieldnormalized citation scores. Retrieved July 29, 2015, from http://dx.doi.org/10.6084/m9.figshare.1496560. 
Haunschild, R., \& Bornmann, L.(2015). Normalization of Mendeley reader counts for impact assessment. Figshare. doi 10.6084/m9.figshare.1334243.

Haunschild, R., Bornmann, L., \& Leydesdorff, L. (2015). Networks of reader and country status: an analysis of Mendeley reader statistics. PeerJ Computer Science, 1, e32. doi 10.7717/peerj-cs.32.

Haustein, S., Costas, R., \& Lariviere, V. (2015). Characterizing Social Media Metrics of Scholarly Papers: The Effect of Document Properties and Collaboration Patterns. Plos One, 10(3). doi: 10.1371/journal.pone.0120495.

Haustein, S., \& Lariviere, V. (2014). A multidimensional analysis of Aslib proceedings - using everything but the impact factor. Aslib Journal of Information Management, 66(4), 358380. doi: 10.1108/ajim-11-2013-0127.

Herranz, N., \& Ruiz-Castillo, J. (2012). Multiplicative and fractional strategies when journals are assigned to several subfields. Journal of the American Society for Information Science and Technology, 63(11), 2195-2205. doi: 10.1002/asi.22629.

Jeng, W., He, D. Q., \& Jiang, J. P. (2015). User Participation in an Academic Social Networking Service: A Survey of Open Group Users on Mendeley. Journal of the Association for Information Science and Technology, 66(5), 890-904. doi: 10.1002/asi.23225.

Kraemer, H. C., Morgan, G. A., Leech, N. L., Gliner, J. A., Vaske, J. J., \& Harmon, R. J. (2003). Measures of clinical significance. Journal of the American Academy of Child and Adolescent Psychiatry, 42(12), 1524-1529. doi: 10.1097/01.chi.0000091507.46853.d1.

Moed, H. F. (2005). Citation analysis in research evaluation. Dordrecht, The Netherlands: Springer.

Mohammadi, E., \& Thelwall, M. (2014). Mendeley Readership Altmetrics for the Social Sciences and Humanities: Research Evaluation and Knowledge Flows. Journal of the Association for Information Science and Technology, 65(8), 1627-1638. doi: 10.1002/asi.23071.

Mohammadi, E., Thelwall, M., \& Kousha, K. (in press). Can Mendeley Bookmarks Reflect Readership? A Survey of User Motivations. Journal of the Association for Information Science and Technology. Journal of the Association for Information Science and Technology. doi: 10.1002/asi.23477.

Priem, J. (2013). Scholarship: Beyond the paper. Nature, 495(7442), 437-440.

Priem, J. (2014). Altmetrics. In B. Cronin \& C. R. Sugimoto (Eds.), Beyond bibliometrics: harnessing multi-dimensional indicators of performance (p. 263-287). Cambridge, MA, USA: MIT Press.

Priem, J., Taraborelli, D., Groth, P., \& Neylon, C. (2010). Altmetrics: a manifesto. Retrieved from Retrieved 28 March 2015, from http://altmetrics.org/manifesto/

Rodgers, E. P., \& Barbrow, S. (2013). A look at altmetrics and its growing significance to research libraries. Ann Arbor, MI, USA: The University of Michigan University Library.

Rodriguez-Navarro, A. (2011). A Simple Index for the High-Citation Tail of Citation Distribution to Quantify Research Performance in Countries and Institutions. Plos One, 6(5). doi: 10.1371/journal.pone.0020510.

Schubert, A., \& Braun, T. (1986). Relative indicators and relational charts for comparative assessment of publication output and citation impact. Scientometrics, 9(5-6), 281-291.

SCImago Reseach Group. (2013). SIR World Report 2013. Granada, Spain: University of Granada. 
Seglen, P. O. (1992). The skewness of science. Journal of the American Society for Information Science, 43(9), 628-638. doi: 10.1002/(sici)1097-4571(199210)43:9<628::aidasi5>3.0.co;2-0.

Thelwall, M., \& Maflahi, N. (2015). Are scholarly articles disproportionately read in their own country? An analysis of Mendeley readers. Journal of the Association for Information Science and Technology, 66(6) 1124-1135. doi: 10.1002/asi.23252.

Torres-Salinas, D., Cabezas-Clavijo, A., \& Jimenez-Contreras, E. (2013). Altmetrics: New Indicators for Scientific Communication in Web 2.0. Comunicar(41), 53-60. doi: 10.3916/c41-2013-05.

van Raan, A. F. J. (2005). Measurement of Central Aspects of Scientific Research: Performance, Interdisciplinarity, Structure. Measurement, 3(1), 19.

Waltman, L., Calero-Medina, C., Kosten, J., Noyons, E. C. M., Tijssen, R. J. W., van Eck, N. J., \& Wouters, P. (2012). The Leiden ranking 2011/2012: Data collection, indicators, and interpretation. Journal of the American Society for Information Science and Technology, 63(12), 2419-2432. doi: 10.1002/asi.22708.

Waltman, L., \& Eck, J. N. V. (2015). Field-normalized citation impact indicators and the choice of an appropriate counting method. Journal of Informetrics, 9(4), 872-894. doi: 10.1016/j.joi.2015.08.001.

Waltman, L., van Eck, N., van Leeuwen, T., Visser, M., \& van Raan, A. (2011a). Towards a new crown indicator: an empirical analysis. Scientometrics, 87(3), 467-481. doi: 10.1007/s11192-011-0354-5

Waltman, L., van Eck, N. J., van Leeuwen, T. N., Visser, M. S., \& van Raan, A. F. J. (2011b). Towards a new crown indicator: some theoretical considerations. Journal of Informetrics, 5(1), 37-47. doi: 10.1016/j.joi.2010.08.001.

Wilsdon, J., Allen, L., Belfiore, E., Campbell, P., Curry, S., Hill, S., Johnson, B. (2015). The Metric Tide: Report of the Independent Review of the Role of Metrics in Research Assessment and Management. doi: 10.13140/RG.2.1.4929.1363.

Zahedi, Z., Costas, R., \& Wouters, P. (2014). Assessing the Impact of Publications Saved by Mendeley Users: Is There Any Different Pattern Among Users? Proceedings of the IATUL Conferences. Paper 4. Retrieved September 10, 2014, from http://docs.lib.purdue.edu/iatul/2014/altmetrics/4

Zahedi, Z., \& Eck, N. J. v. (2014). Visualizing readership activity of Mendeley users using VOSviewer. Retrieved July 8, 2014, from http://figshare.com/articles/Visualizing_readership_activity_of_Mendeley_users_using_V OSviewer/1041819

Zitt, M., \& Small, H. (2008). Modifying the journal impact factor by fractional citation weighting: The audience factor. Journal of the American Society for Information Science and Technology, 59(11), 1856-1860. doi: 10.1002/asi.20880. 


\section{Appendix}

Table A.1: WoS subject categories and their two-letter codes with the average Mendeley reader counts for articles and reviews (ordered alphabetically by WoS subject category)

\begin{tabular}{|c|c|c|c|}
\hline \multirow[t]{2}{*}{ WoS subject category } & \multirow[t]{2}{*}{ Code } & \multicolumn{2}{|c|}{$\begin{array}{c}\text { Average no. of readers per } \\
\text { paper }\end{array}$} \\
\hline & & Articles & Reviews \\
\hline Acoustics & aa & 5.84 & 9.48 \\
\hline Agricultural Economics, \& Policy & af & 8.83 & 27.20 \\
\hline Agricultural Engineering & ae & 13.88 & 52.62 \\
\hline Agriculture, Dairy \& Animal Science & $\mathrm{ad}$ & 4.90 & 12.88 \\
\hline Agriculture, Multidisciplinary & ah & 7.01 & 16.35 \\
\hline Agronomy & am & 7.18 & 22.41 \\
\hline Allergy & aq & 7.05 & 9.23 \\
\hline Anatomy \& Morphology & ay & 6.27 & 16.88 \\
\hline Andrology & az & 3.09 & 10.07 \\
\hline Anesthesiology & ba & 7.52 & 14.44 \\
\hline Anthropology & bf & 13.16 & 14.87 \\
\hline Archaeology & bi & 8.95 & 13.33 \\
\hline Architecture & bk & 2.97 & 3.00 \\
\hline Area Studies & $\mathrm{bm}$ & 4.28 & 3.94 \\
\hline Art & $\mathrm{bp}$ & 3.32 & 2.20 \\
\hline Asian Studies & or & 1.69 & 1.67 \\
\hline Astronomy \& Astrophysics & bu & 4.85 & 14.22 \\
\hline Audiology \& Speech-Language Pathology & $\mathrm{cl}$ & 9.58 & 17.39 \\
\hline Automation \& Control Systems & $\mathrm{ac}$ & 4.36 & 13.55 \\
\hline Behavioral Sciences & $\mathrm{cn}$ & 15.22 & 55.16 \\
\hline Biochemical Research Methods & co & 13.73 & 26.75 \\
\hline Biochemistry \& Molecular Biology & $\mathrm{cq}$ & 12.42 & 25.38 \\
\hline Biodiversity Conservation & bd & 25.24 & 53.08 \\
\hline
\end{tabular}




\begin{tabular}{|c|c|c|c|}
\hline Biology & $\mathrm{cu}$ & 14.14 & 38.20 \\
\hline Biophysics & da & 8.65 & 23.31 \\
\hline Biotechnology \& Applied Microbiology & $\mathrm{db}$ & 12.76 & 32.08 \\
\hline Business & di & 24.13 & 43.77 \\
\hline Business, Finance & $\mathrm{dk}$ & 13.66 & 20.83 \\
\hline Cardiac \& Cardiovascular System & $\mathrm{dq}$ & 5.98 & 12.62 \\
\hline Cell \& Tissue Engineering & $\mathrm{ct}$ & 12.18 & 31.50 \\
\hline Cell Biology & $\mathrm{dr}$ & 16.48 & 32.41 \\
\hline Chemistry, Analytical & ea & 5.23 & 21.09 \\
\hline Chemistry, Applied & $\mathrm{dw}$ & 5.46 & 12.81 \\
\hline Chemistry, Inorganic \& Nuclear & ec & 3.57 & 11.35 \\
\hline Chemistry, Medicinal & $\mathrm{dx}$ & 5.10 & 13.58 \\
\hline Chemistry, Multidisciplinary & dy & 10.15 & 28.48 \\
\hline Chemistry, Organic & ee & 4.60 & 11.29 \\
\hline Chemistry, Physical & ei & 8.66 & 30.29 \\
\hline Classics & eo & 0.55 & 0.33 \\
\hline Clinical Neurology & $\mathrm{rt}$ & 7.44 & 13.14 \\
\hline Communication & $\mathrm{eu}$ & 12.67 & 17.29 \\
\hline Computer Science, Artificial Intelligence & ep & 9.58 & 23.69 \\
\hline Computer Science, Cybernetics & er & 11.45 & 12.22 \\
\hline Computer Science, Hardware \& Architecture & es & 6.21 & 53.22 \\
\hline Computer Science, Information Systems & et & 9.08 & 25.80 \\
\hline Computer Science, Interdisciplinary Applications & ev & 8.88 & 26.50 \\
\hline Computer Science, Software Engineering & ew & 8.31 & 43.30 \\
\hline Computer Science, Theory \& Methods & ex & 7.13 & 42.27 \\
\hline Construction \& Building Technology & fa & 7.93 & 24.75 \\
\hline Criminology \& Penology & $\mathrm{fe}$ & 7.15 & 16.24 \\
\hline Critical Care Medicine & ds & 9.96 & 17.13 \\
\hline Crystallography & fi & 2.85 & 8.71 \\
\hline Cultural Studies & en & 5.25 & 4.00 \\
\hline
\end{tabular}


Dance

fs

1.46

0.00

Demography

8.27

11.56

Dentistry, Oral Surgery \& Medicine

9.18

14.03

Dermatology

3.86

8.49

Developmental Biology

13.57

30.96

Ecology

25.61

76.02

Economics

10.03

16.07

Education \& Educational Research

12.48

25.13

Education, Scientific Disciplines

ha

9.53

13.94

Education, Special

9.50

17.03

Electrochemistry

hq

6.39

26.16

Emergency Medicine

5.78

10.68

Endocrinology \& Metabolism

ff

ia

7.15

17.15

Energy \& Fuels

11.04

31.13

Engineering, Aerospace

2.85

11.19

Engineering, Biomedical

9.34

25.23

Engineering, Chemical

5.88

26.36

Engineering, Civil

6.41

23.91

Engineering, Electrical \& Electronic

4.82

16.09

Engineering, Environmental

9.32

28.13

Engineering, Geological

4.33

4.33

Engineering, Industrial

11.00

26.64

Engineering, Manufacturing

6.67

17.34

Engineering, Marine

3.00

4.50

Engineering, Mechanical

3.82

20.11

Engineering, Multidisciplinary

4.24

9.38

Engineering, Ocean

4.26

5.00

Engineering, Petroleum

3.77

4.83

Entomology

5.65

20.34

Environmental Sciences

11.33

28.60 


\begin{tabular}{|c|c|c|c|}
\hline Environmental Studies & $\mathrm{jb}$ & 18.46 & 26.99 \\
\hline Ergonomics & $\mathrm{ji}$ & 14.34 & 15.14 \\
\hline Ethics & hf & 10.05 & 8.19 \\
\hline Ethnic Studies & $\mathrm{jm}$ & 7.56 & 4.73 \\
\hline Evolutionary Biology & ht & 27.94 & 70.39 \\
\hline Family Studies & jo & 9.34 & 15.63 \\
\hline Film, Radio, \& Television & js & 3.75 & 6.50 \\
\hline Fisheries & $\mathrm{ju}$ & 7.75 & 22.47 \\
\hline Folklore & $\mathrm{jw}$ & 0.86 & 0.00 \\
\hline Food Science \& Technology & jy & 6.09 & 15.88 \\
\hline Forestry & $\mathrm{ka}$ & 10.84 & 22.08 \\
\hline Gastroenterology \& Hepatology & ki & 4.75 & 9.55 \\
\hline Genetics \& Heredity & $\mathrm{km}$ & 15.27 & 48.74 \\
\hline Geochemistry \& Geophysics & $\mathrm{gc}$ & 7.13 & 23.48 \\
\hline Geography & $\mathrm{ku}$ & 14.29 & 15.20 \\
\hline Geography, Physical & $\mathrm{kv}$ & 15.26 & 38.23 \\
\hline Geology & ky & 7.91 & 12.59 \\
\hline Geosciences, Multidisciplinary & le & 9.36 & 22.09 \\
\hline Geriatrics \& Gerontology & li & 8.33 & 15.92 \\
\hline Gerontology & $\mathrm{lj}$ & 7.83 & 10.85 \\
\hline Health Care Sciences \& Services & $\mathrm{hl}$ & 9.77 & 14.38 \\
\hline Health Policy \& Services & lq & 9.34 & 14.83 \\
\hline Hematology & $\mathrm{ma}$ & 7.51 & 14.57 \\
\hline History & $\mathrm{mm}$ & 1.81 & 1.50 \\
\hline History \& Philosophy of Science & $\mathrm{mq}$ & 6.57 & 6.52 \\
\hline History of Social Sciences & $\mathrm{mr}$ & 3.59 & 1.22 \\
\hline Horticulture & $\mathrm{mu}$ & 7.01 & 19.89 \\
\hline Hospitality, Leisure, Sport, \& Tourism & $\mathrm{mw}$ & 13.55 & 33.25 \\
\hline Humanities, Multidisciplinary & $\mathrm{bq}$ & 2.39 & 4.96 \\
\hline Imaging Science \& Photographic Technology & ue & 9.98 & 40.45 \\
\hline
\end{tabular}




\begin{tabular}{|c|c|c|c|}
\hline Immunology & ni & 8.30 & 21.51 \\
\hline Industrial Relations \& Labor & $\mathrm{nm}$ & 8.65 & 16.10 \\
\hline Infectious Diseases & $\mathrm{nn}$ & 7.48 & 13.37 \\
\hline Information Science \& Library Science & $\mathrm{nu}$ & 17.48 & 23.67 \\
\hline Instruments \& Instrumentation & oa & 3.91 & 18.68 \\
\hline Integrative \& Complementary Medicine & oi & 5.64 & 10.92 \\
\hline International Relations & oe & 9.45 & 6.33 \\
\hline Language \& Linguistics Theory & oy & 5.29 & 5.03 \\
\hline Law & om & 4.93 & 4.37 \\
\hline Limnology & $\mathrm{ou}$ & 8.77 & 25.14 \\
\hline Linguistics & ot & 8.62 & 15.87 \\
\hline Literary Reviews & $\mathrm{oz}$ & 3.09 & 0.00 \\
\hline Literary Theory \& Criticism & ox & 3.41 & 0.00 \\
\hline Literature & pa & 1.07 & 0.27 \\
\hline Literature, African, Australian, \& Canadian & $\mathrm{pd}$ & 0.72 & 0.00 \\
\hline Literature, American & $\mathrm{pf}$ & 2.45 & 0.67 \\
\hline Literature, British Isles & $\mathrm{pg}$ & 0.26 & 0.00 \\
\hline Literature, German, Dutch, \& Scandinavian & $\mathrm{ph}$ & 0.60 & 0.00 \\
\hline Literature, Romance & $\mathrm{qc}$ & 0.78 & 0.50 \\
\hline Literature, Slavic & $\mathrm{qd}$ & 0.38 & 0.00 \\
\hline LOGIC & $\mathrm{q} 1$ & 1.97 & 0.00 \\
\hline Management & $\mathrm{pc}$ & 21.22 & 39.42 \\
\hline Marine \& Freshwater Biology & pi & 10.94 & 28.65 \\
\hline Materials Science, Biomaterials & qe & 9.85 & 36.75 \\
\hline Materials Science, Ceramics & $\mathrm{pk}$ & 3.49 & 8.98 \\
\hline Materials Science, Characterization, Testing & qf & 3.08 & 7.23 \\
\hline Materials Science, Coatings \& Films & qg & 4.69 & 9.19 \\
\hline Materials Science, Composites & qh & 5.19 & 25.48 \\
\hline Materials Science, Multidisciplinary & $\mathrm{pm}$ & 7.83 & 29.75 \\
\hline Materials Science, Paper \& Wood & $\mathrm{pj}$ & 3.58 & 11.78 \\
\hline
\end{tabular}


Materials Science, Textiles

qj

3.63

11.58

Mathematical \& Computational Biology

$\mathrm{mc}$

18.42

38.54

Mathematics

$\mathrm{pq}$

0.92

0.76

Mathematics, Applied

pn

1.67

3.42

Mathematics, Interdisciplinary Applications

po

3.94

8.90

Mechanics

4.00

16.42

Medical Ethics

$\mathrm{pu}$

6.31

7.86

Medical Informatics

9.29

26.60

Medical Laboratory Technology

3.72

9.66

Medicine, General \& Internal

7.24

10.46

Medicine, Legal

6.46

7.99

Medicine, Research \& Experimental

8.30

18.20

Medieval \& Renaissance Studies

qa

0.75

0.00

Metallurgy \& Metallurgical Engineering

3.34

9.81

Meteorology \& Atmospheric Sciences

pz

8.95

24.41

Microbiology

9.77

29.05

Microscopy

5.55

10.32

Mineralogy

4.08

9.91

Mining \& Mineral Processing

3.61

8.33

Multidisciplinary Sciences

22.73

47.08

Music

4.24

1.80

Mycology

6.50

19.45

Nanoscience \& Nanotechnology

10.82

33.66

Neuroimaging

23.13

72.67

Neurosciences

rx

15.60

40.51

Nuclear Science \& Technology

2.44

6.35

Nursing

ry

5.90

9.76

Nutrition \& Dietetics

8.24

16.80

Obstetrics \& Gynecology

4.45

8.15

Oceanography

10.03

27.78 


\begin{tabular}{|c|c|c|c|}
\hline Oncology & $\mathrm{dm}$ & 6.32 & 14.35 \\
\hline Operations Research \& Management Science & pe & 8.83 & 28.07 \\
\hline Ophthalmology & su & 5.09 & 7.39 \\
\hline Optics & sy & 4.60 & 29.06 \\
\hline Ornithology & ta & 12.92 & 32.93 \\
\hline Orthopedics & tc & 6.97 & 10.66 \\
\hline Otorhinolaryngology & $\mathrm{td}$ & 3.63 & 5.90 \\
\hline Paleontology & te & 6.53 & 14.78 \\
\hline Parasitiology & $\mathrm{ti}$ & 11.22 & 26.70 \\
\hline Pathology & $\mathrm{tm}$ & 4.64 & 12.46 \\
\hline Pediatrics & tq & 5.08 & 8.94 \\
\hline Peripheral Vascular Diseases & $\mathrm{zd}$ & 6.35 & 11.82 \\
\hline Pharmacology \& Pharmacy & tu & 5.66 & 18.13 \\
\hline Philosophy & ua & 3.82 & 3.55 \\
\hline Physics, Applied & $\mathrm{ub}$ & 6.58 & 30.94 \\
\hline Physics, Atomic, Molecular, \& Chemical & uh & 6.18 & 15.29 \\
\hline Physics, Condensed Matter & uk & 8.09 & 31.48 \\
\hline Physics, Fluids \& Plasmas & uf & 4.89 & 27.87 \\
\hline Physics, Mathematical & ur & 3.99 & 7.02 \\
\hline Physics, Multidisciplinary & ui & 6.82 & 20.04 \\
\hline Physics, Nuclear & un & 2.59 & 3.32 \\
\hline Physics, Particles \& Fields & up & 3.10 & 3.80 \\
\hline Physiology & um & 8.12 & 20.33 \\
\hline Planning \& Development & uq & 15.42 & 25.38 \\
\hline Plant Sciences & de & 9.57 & 32.14 \\
\hline Poetry & ut & 0.22 & 0.00 \\
\hline Political Science & $\mathrm{uu}$ & 8.76 & 8.08 \\
\hline Polymer Science & uy & 5.23 & 23.39 \\
\hline Primary Health Care & $\mathrm{ml}$ & 6.81 & 13.02 \\
\hline Psychiatry & ve & 10.10 & 16.94 \\
\hline
\end{tabular}




\begin{tabular}{|c|c|c|c|}
\hline Psychology & vi & 14.54 & 45.97 \\
\hline Psychology, Applied & nq & 17.03 & 38.66 \\
\hline Psychology, Biological & bv & 15.52 & 40.98 \\
\hline Psychology, Clinical & eq & 11.48 & 21.10 \\
\hline Psychology, Developmental & my & 14.68 & 22.65 \\
\hline Psychology, Educational & hi & 14.04 & 30.04 \\
\hline Psychology, Experimental & $\mathrm{vx}$ & 19.06 & 85.22 \\
\hline Psychology, Mathematical & vs & 13.10 & 28.85 \\
\hline Psychology, Multidisciplinary & $\mathrm{vj}$ & 16.12 & 41.02 \\
\hline Psychology, Psychoanalysis & $\mathrm{vp}$ & 3.38 & 3.38 \\
\hline Psychology, Social & wq & 17.28 & 27.71 \\
\hline Public, Administration & $\mathrm{vm}$ & 9.10 & 12.27 \\
\hline Public, Environmental \& Occupational Health & ne & 7.68 & 14.39 \\
\hline Radiology, Nuclear Medicine, \& Medical Imaging & vy & 8.63 & 23.25 \\
\hline Rehabilitation & $\mathrm{wc}$ & 9.12 & 14.39 \\
\hline Religion & yi & 2.19 & 6.22 \\
\hline Remote Sensing & $\mathrm{sr}$ & 9.67 & 43.91 \\
\hline Reproductive Biology & wf & 5.30 & 12.07 \\
\hline Respiratory System & we & 6.06 & 9.72 \\
\hline Rheumatology & wh & 6.58 & 10.87 \\
\hline Robotics & $\mathrm{rb}$ & 8.61 & 22.73 \\
\hline Social Issues & wm & 7.23 & 17.32 \\
\hline Social Sciences, Biomedical & wV & 10.51 & 18.12 \\
\hline Social Sciences, Interdisciplinary & wu & 10.56 & 16.84 \\
\hline Social Sciences, Mathematical Methods & ps & 7.54 & 25.80 \\
\hline Social Work & wy & 7.20 & 16.14 \\
\hline Sociology & xa & 10.67 & 11.74 \\
\hline Soil Science & $\mathrm{xe}$ & 7.93 & 21.91 \\
\hline Spectroscopy & $\mathrm{xq}$ & 3.68 & 12.31 \\
\hline Sport Sciences & $\mathrm{xw}$ & 11.44 & 15.54 \\
\hline
\end{tabular}




\begin{tabular}{llcc} 
Statistics \& Probability & xy & 4.42 & 13.10 \\
Substance Abuse & gm & 7.49 & 14.72 \\
Surgery & ya & 4.45 & 6.90 \\
Telecommunications & ye & 4.21 & 14.33 \\
Theater & yg & 1.40 & 0.00 \\
Thermodynamics & dt & 5.28 & 34.88 \\
Toxicology & yo & 5.68 & 13.46 \\
Transplantation & yp & 4.26 & 7.59 \\
Transportation & yq & 13.89 & 12.12 \\
Transportation Science \& Technology & yr & 6.05 & 9.87 \\
Tropical Medicine & yu & 10.82 & 23.13 \\
Urban Studies & yy & 14.21 & 15.21 \\
Urology \& Nephrology & za & 3.88 & 7.44 \\
Veterinary Sciences & zc & 7.33 & 14.98 \\
Virology & ze & 8.93 & 16.84 \\
Water Resources & zr & 6.93 & 23.29 \\
Women's Studies & zk & 6.97 & 5.68 \\
Zoology & zm & 12.81 & 23.90 \\
\hline
\end{tabular}

